\title{
Paraumbilical collateral veins on MRI as possible protection against portal venous thrombosis in candidates for liver transplantation
}

\author{
Sachit Verma \\ Thomas Jefferson University \\ Donald G. Mitchell \\ Thomas Jefferson University \\ Yulia Lakhman \\ Thomas Jefferson University \\ Diane Bergin

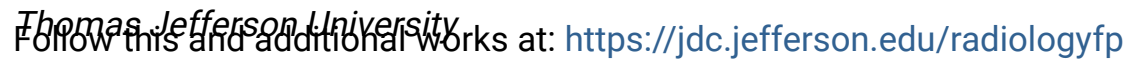 \\ riradet df Emelinadiology Commons \\ Theras is feferso Uwiversitw access to this document benefits you
}

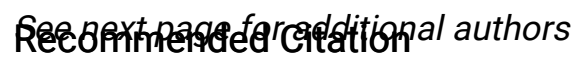

Verma, Sachit; Mitchell, Donald G.; Lakhman, Yulia; Bergin, Diane; Dolin, Ronald J.; Doria, Cataldo; and Parker, Laurence, "Paraumbilical collateral veins on MRI as possible protection against portal venous thrombosis in candidates for liver transplantation" (2007). Department of Radiology Faculty Papers. Paper 6.

https://jdc.jefferson.edu/radiologyfp/6

This Article is brought to you for free and open access by the Jefferson Digital Commons. The Jefferson Digital Commons is a service of Thomas Jefferson University's Center for Teaching and Learning (CTL). The Commons is a showcase for Jefferson books and journals, peer-reviewed scholarly publications, unique historical collections from the University archives, and teaching tools. The Jefferson Digital Commons allows researchers and interested readers anywhere in the world to learn about and keep up to date with Jefferson scholarship. This article has been accepted for inclusion in Department of Radiology Faculty Papers by an authorized administrator of the Jefferson Digital Commons. For more information, please contact: JeffersonDigitalCommons@jefferson.edu. 


\section{Authors}

Sachit Verma, Donald G. Mitchell, Yulia Lakhman, Diane Bergin, Ronald J. Dolin, Cataldo Doria, and Laurence Parker 


\section{Paraumbilical collateral veins on MRI as possible protection}

\section{against portal venous thrombosis in candidates for liver}

\section{transplantation}

Sachit K. Verma, Donald G. Mitchell, ${ }_{1}^{1}$ Yulia Lakhman, Diane Bergin,

Ronald J. Dolin, Cataldo Doria, Laurence Parker ${ }^{1}$

${ }^{1}$ Department of Radiology

Thomas Jefferson University Hospital

132 South 10th St, 1094 Main Building

Philadelphia, PA 19107, USA

2 Department of Surgery

Thomas Jefferson University Hospital

Philadelphia, PA, USA

Correspondence to: Donald G. Mitchell; email: donald.mitchell@ jefferson.edu

\section{Abstract}

Background: We retrospectively evaluate the potential protective influence of patent paraumblical vein (PUV) collaterals against portal vein (PV) thrombosis and reduced PV diameter in candidates for orthotopic liver transplant (OLT)

Methods: Dynamic 3D contrast-enhanced MRI at 1.5T was obtained in 309 patients with cirrhosis without evidence of malignancy. All MR studies were reviewed by one reader for PUV collaterals, PV thrombosis and PV diameter. Statistical analysis was performed by Fisher exact tests; 50 selected studies were reviewed independently by two additional readers to determine interobserver agreement via intraclass correlation coefficient (ICC). 
Results: Patent PUV was noted in 119 of 309 patients (38.5\%). Mean PV diameter was $13.4 \pm 3.0 \mathrm{~mm}$ in patients with PUV compared with $11.3 \pm 3.6 \mathrm{~mm}$ without PUV $(P<0.01)$. Main $P V$ thrombosis was present in 13 of 309 patients $(4.2 \%)$ and significantly more frequent in those without PUV than with PUV $(6.3 \%$ vs. $0.8 \%, \mathrm{P}<$ 0.05). ICC indicated almost perfect agreement among three readers for presence of PUV collaterals $(I C C=0.91)$ and PV thrombosis $(I C C=0.96)$.

Conclusion: Our results suggest that patients with patent PUV appear less likely to develop main PV thrombosis or small PV diameter, suggesting a protective effect of PUV on PV patency.

Key words: Paraumbilical vein-Cirrhosis—Portal vein thrombosis-MRI-liver transplantation

Cirrhosis of the liver is characterized by progressive fibrosis and architectural disarrangement, leading to an increased intrahepatic resistance to portal venous inflow and the development of portal hypertension. This is exacerbated by an increase in portal blood flow, promoted by splanchnic arteriolar vasodilatation and splenomegaly [1]. The pathological increase in portal pressure leads to the development of a collateral portal-systemic circulation, which forms in an attempt to decompress the portal system by diverting blood flow to systemic veins [2]. This in turn decreases portal vein flow volume and velocity, which is probably a factor in the 
high incidence of portal vein (PV) thrombosis among patients with portal hypertension [3-5].

Liver transplantation is sometimes needed to treat hepatic failure or early stage hepatocellular carcinoma. Adequate portal venous inflow to the hepatic allograft is mandatory for successful liver transplantation [6]. PV thrombosis or small PV caliber less than $1 \mathrm{~cm}$ while not an absolute contraindication to orthotopic liver transplantation (OLT), has a substantial effect on surgical complexity and perioperative morbidity and mortality rates [7]. To properly plan for OLT and to minimize operative time, preoperative detection of $\mathrm{PV}$ thrombosis is of paramount importance. Color flow Doppler sonography and conventional angiography are commonly used to study the portal venous system, but each has its limitations. For this reason, regular short-interval serial MRI is recommended for candidates for hepatic transplantation, to direct intraopertive visualization [8-10].

A subset of patients with portal hypertension shunt their portosystemic flow primarily through paraumbilical vein (PUV) collaterals, which drain blood from the left PV toward veins of the ventral abdominal wall [11]. This portosystemic shunt is different from others, in that it involves shunting through the main PV, rather than away from it. We are not aware of any data to support the theoretical protective effect of patent PUV against PV thrombosis and reduced PV size. Thus we retrospectively evaluate the potential protective influence of PUV collaterals against PV thrombosis and reduced PV diameter in candidates for OLT.

Material and methods 
Patients

This HIPAA compliant retrospective study was approved by our institutional review board. Informed consent was not required. Liver MR records and clinical MR requests from January 1999 through January 2005 at our institution were searched and then cross-referenced to the histopathology records to identify patients with histopathologically proved cirrhosis. Patients were excluded from the study (a) if they had any evidence of solid malignant tumors [primary HCC $(n=68)$, primary pancreaticobiliary cancer $(n=12)$, secondary malignancy (metastasis from pancreatic cancer $n=9$, colorectal carcinoma $n=3$, renal carcinoma $n=3$ ] (b) cirrhosis without histopathological confirmation $(n=17)$, transjugular intrahepatic portosystemic shunt (TIPS) $(n=2)$. Consequently the study population included 309 consecutive patients (191 men and 118 women) aged 18-86 years (mean age 54 years). These patients had been referred for MR imaging to evaluate the severity of cirrhosis and portal hypertension and to acquire preoperative studies before liver transplantation. All patients had biopsy-proven cirrhosis and documented portal hypertension proven by endoscopic visualization of varices or established imaging criteria [12]. The cirrhosis was attributed to viral hepatitis alone in 232 patients (HCV $n=170, \mathrm{HBV} n=52$, both $n=10$ ), alcohol in 48 , cryptogenic in 12 , primary sclerosing cholangitis in seven, autoimmune in three, primary biliary cirrhosis in three, non alcoholic steatohepatitis in two, congenital biliary atresia in remaining two patients.

Cirrhosis was histopathologically confirmed by means of percutaneous liver 
biopsy in 280 patients and transjugular liver biopsy in 29. According to Child-Pugh classification, of the 309 patients, 93 were in class A, 147 in class B and 69 in class C.

MR Imaging

All MR examinations were performed during suspended respiration with a 1.5-T system and phased array coil (Signa: General Electric Medical Systems, Milwaukee, WI; or Achieva: Philips, Best, Netherlands). The sequences included twodimensional (2D) coronal and axial single-shot fast spin-echo T2-weighted MR images (TR infinite; TE 180-200 ms); axial fat suppressed fast spin-echo T2weighted MR images (TR 2500-4000 ms, TE 70-90 ms); spoiled dual gradient echo (SGRE) T1weighted in-and out-of-phase MR images (TR 120-200 ms, TE = 2.3 and $4.6 \mathrm{~ms}$, flip angle $90^{\circ}$ ). Parameters for $2 \mathrm{D}$ images included section thickness $5-$ $8 \mathrm{~mm}$ with intersection gap 0-1 mm; matrix, $256 \cdot 160-192$; field of view $32 \mathrm{~cm}$ transverse and $24 \mathrm{~cm}$ anteroposterior; NSA $=1$ or less. Three-dimensional (3D) dynamic enhanced transverse SGRE fat suppressed MR images were obtained with $5 \mathrm{~mm}$ thick resolution in the slice axis, at $2.2-2.5 \mathrm{~mm}$ increments using zero-fill interpolation, TR/ TE/flip angle = 3-6.1/0.9-2.1/12-20을 , and parameters otherwise similar to those of the 2D images. Acquisition time per 3D volume was $21 \mathrm{~s}$, obtained during suspended respiration; $20 \mathrm{cc}$ of contrast material (Magnevist; Berlex Laboratories, Wayne, NJ, USA) was administered intravenously via power-injector (OptistarTM LE; Mallinckrodt; Hazelwood, MO, USA) at $2 \mathrm{~mL} / \mathrm{s}$ followed by a $20-\mathrm{mL}$ saline solution flush. First-pass arterial enhancement was optimized using a timing 
bolus sequence, or by observing enhancement on images reconstructed real-time. Dynamic imaging was performed during breath-hold before the injection (unenhanced), immediately after injection (hepatic arterial phase) and $30 \mathrm{~s}$ afterward (portal venous phase). Additional delayed phase images of the entire liver were acquired using a 2D single-section SGRE technique with TR/TE of 19-20/ 1.5-2.1

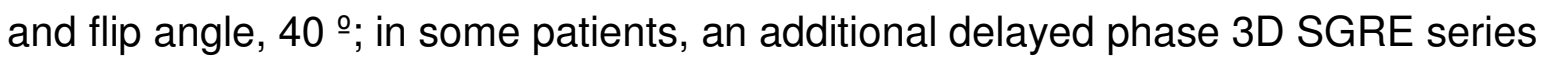
was acquired.

Review of records and images MR images were reviewed independently on a picture archiving and communication system (PACS) workstation (Canon Medical Systems, Irvine, CA, USA) initially by one radiologist (S.K.V with 3 years of body MR experience), without access to the prospective reports or any clinical information.

All patients were screened for the presence of portosystemic shunts and varices such as splenorenal shunt, esophageal varices with particular attention to PUV that runs along the ligamentum teres in the falciform ligament. Based on the retrospective image review, two groups from within the study population, one with and one without patent PUV were identified. Patent PUV was defined as a clearly visible vessel connecting the left PV to collateral veins at the anterior abdominal wall. Maximum transverse diameters of PUV at its widest part were recorded on 3D gadolinium-enhanced GRE fat suppressed sequence during portal venous phase.

Thrombosis was classified as occlusive or nonocclusive. The maximum transverse diameter of the main PV was measured with calipers from wall to wall 
midway between the splenoportal confluence and the portal vein bifurcation in the porta hepatis on 3D gadolinium-enhanced GRE fat suppressed sequence during portal venous phase. The splenic vein was evaluated from the confluence to splenic hilum; and the superior mesenteric vein, from the confluence to the right colic branch. The vessels were defined as patent if the entire lumen was filled with contrast material—enhanced blood on enhanced images. An occlusive thrombus was defined as a nonenhancing filling defect within the lumen of the vessel seen on contrast enhanced images. Nonocclusive thrombi were identified when there was contrast-opacified blood adjacent to the existing thrombus, and these thrombi were further divided into those occupying more than $50 \%$ of the lumen or less than $50 \%$ of the lumen. A splenorenal shunt was defined as a spontaneous anastomosis of the splenic vein or a perisplenic varix to an enlarged left renal vein. Presence or absence of ascites was noted.

To evaluate interobserver agreement, a subset of 50 examinations were reviewed independently by two additional readers (D.G.M., Y.L with 21. 2 years of body MRI experience) for assessment of PV thrombosis and PUV, who were blinded to the initial evaluation by the first reader. These 50 examinations included all patients with main or branch PV thrombus identified by the first reader $(n=17)$, and 33 arbitrarily selected additional examinations from both PUV and non-PUV groups. Data were entered into a spreadsheet (Excel; Microsoft, Redmond, WA, USA). When the readers disagreed about the presence of $\mathrm{PV}$ thrombosis, a majority opinion was used as final decision for data analyses. 
Statistical evaluation

Statistical analysis was performed with SAS software (SAS Institute, Cary, NC, USA). Descriptive statistics (mean, SD) were provided where appropriate. A nonpaired Student $t$ test was used to assess the statistical significance of diameter of main PV between patients with vs. without patent PUV. We used a Fisher exact test to determine the statistical significance of differences among the patients with and without PUV in incidence of main PV thrombosis, occluded main PV thrombosis and main PV size less than $1 \mathrm{~cm}$. Reliability for the presence PV thrombosis and PUV collaterals for the three readers was assessed by computing the intraclass correlation coefficient (ICC). ICC for inter-observer reliability was performed using Shrout-Fleiss methodology [13].

Results

Results are summarized in Table 1. Main PV thrombosis was present in 13 of 309 (4.2\%) patients; main PV thrombosis was occlusive in six and nonocclusive in seven. For all 13 patients with main PV thrombus, the blinded retrospective detection of presence and extent of thrombus agreed with the findings in the prospective report. Multireader discrepancy in interpreting the presence of thrombus in main PV occurred in only 1 of 13 patients with partial PVT, which was resolved in consensus as presence of PV thrombosis. Interobserver ICC for main PV thrombosis was 0.96. Main PV thrombus extended to the confluence of the splenic and superior mesenteric veins in two patients. No patients had thrombosis of the 
splenic vein, superior mesenteric vein or confluence without involvement of the main PV. Four patients without main PV thrombus had branch thrombi identified. These four examinations were included among the 50 reviewed by additional readers, but thrombi that did not involve the main PV were not considered further.

Splenorenal shunt was recognized in 24 (8\%) of 309 patients including 5 of 119 (4.2\%) with PUV and 19 of 190 (10\%) patients without PUV $(\mathrm{P}<0.05)$.

Patent PUV was noted in 119 of 309 patients (38.5\%) (group 1), with a mean diameter of $5.2 \pm 3.3 \mathrm{~mm}$ (range $2-19 \mathrm{~mm}$ ). The prevalence of PUV were more often present in patients with Child-Pugh class C (52\%)or class B clinical status (41\%) than in those with class A status $(24 \%)(P<0.05)$.

For the subset of 50 patients viewed by three readers, interobserver ICC for PUV collaterals was 0.91 . PUV was not present in any of the six patients who had occlusive main $\mathrm{PV}$ thrombosis $(\mathrm{P}=0.08)$ (Fig. 1). Overall, main $\mathrm{PV}$ thrombosis which was nonocclusive seen only in 1 of 119 (0.8\%) patent PUV (Fig. 2), compared with 12 of the $190(6.3 \%)$ without PUV $(P=0.02)$. Mean PV diameter was $13.4 \pm 3.0$ $\mathrm{mm}$ among the 119 patients with PUV (Fig. 3) compared with $11.3 \pm 3.6 \mathrm{~mm}$ among the 190 patients without PUV (group 2) $(\mathrm{P}<0.01)$. PV diameter size less than $1 \mathrm{~cm}$ was seen in 10 of 190 (5.1\%) patients without PUV, compared with 3 of 119 (2.5\%) patients with PUV $(P=0.19)$. Three of these patients with PUV who had PV less than $1 \mathrm{~cm}$ had splenorenal shunt (diameters 8,10 and $20 \mathrm{~mm}$; mean $12.6 \pm 6.4 \mathrm{~mm}$ ) (Fig. 4), none had ascites.

Esophageal varices were seen in 57 of 309 (18\%) patients, including 26 of 119 (22\%) with PUV and 31 of 190 (16\%) patients without PUV $(P=N S)$. 
Discussion

The presence and hemodynamic effects of patent PUV in patients with portal hypertension have been examined with Doppler sonography and MRI [8-10, 14], with reported frequency of PUV ranging from 11 to $97 \%$ [11, 15-17]. In our study, we detected PUV in $38 \%$ of MRI examinations in the patients with portal hypertension. The increasing prevalence of PUV collaterals with progression of liver disease was evident in the present study, in which shunts were found significantly more frequently in patients with grade $\mathrm{C}$ or B cirrhosis than in those with grade $\mathrm{A}$ cirrhosis [18].

The PV diameter, averaging $1 \mathrm{~cm}$ in healthy adults [19], increases during the initial stages of portal hypertension [12, 20], with a PV larger than $13 \mathrm{~mm}$ reported as characteristic of portal hypertension in the appropriate clinical setting [21]. However, massive shunting away from the liver in later stages is associated with reduced PV caliber or with portal thrombosis, both of which can complicate OLT, particularly if not detected preoperatively [14, 22]. The incidence of PV thrombosis varies according to the characteristics of the patients evaluated, ranging from 0.6 to $16.6 \%$ [23-26]. Therefore, in our clinical practice, we routinely evaluate the patency of the PV preoperatively, using frequent sonography and annual MRI, to determine if an alternative surgical method is necessary to assure portal inflow.

Ability to detect PUV collaterals is important in diagnosing and managing patients with chronic liver disease and portal hypertension. It is necessary for transplant surgeon to know the extend and patency of PUV collaterals as it may increase the 
risk of bleeding if it is occluded due to elevated portal pressure. Although portal hypertension decreases flow to hepatic parenchyma regardless of the character of portosystemic shunting, a PUV allows this shunting to occur through the main PV, rather than away from it, thereby maintaining the volume and velocity of main PV flow, potentially preventing reduced main PV size and reducing the likelihood of main PV thrombosis [27]. In contrast, in the presence of a large splenorenal shunt, a portion of splenic venous blood is diverted into the shunt and subsequently causes a significant reduction of portal venous velocity and flow volume [28-30]. Our observations have confirmed the expected protective effect of PUV against complete PV thrombosis consistent with previous observations [31] of higher PV mean velocity in the presence of a patent PUV. However the magnitude of this protective effect may be reduced if the PUV is small, or if massive extrahepatic (e.g. splenorenal) shunt develops, as in all 3 of 119 patients with PUV who had PV size less than $1 \mathrm{~cm}$ or if ascites is present [32], as in 1 of 119 patient with PUV who had non occlusive thrombus in main PV.

The potential protective effect of PUV on maintaining PV diameter is supported by our finding of larger PV size in patients with PUV compared to those without PUV. However, the incidence of portal vein smaller than $1 \mathrm{~cm}$ was not significantly different between these two groups, possibly because of variable contribution towards portosystemic shunting of splenorenal and other shunts. Another potential protective effect of PUV, against bleeding from esophageal varices, cannot be directly addressed from our study.

Our study has several limitations. One limitation was our inability to confirm our 
imaging findings by conventional direct or indirect portographic methods.

Conventional portography is invasive and therefore was not performed in most patients. Many investigators have reported the use of Doppler sonography [27, 33]to determine the direction of portal flow. In our experience, we have noted some problems, including body habitus, operator dependence, failure to depict partial thrombosis or shunts, and the false positive diagnosis of PV thrombosis due to stagnant or hepatofugal flow. Any of a number of technical factors (such as inappropriate color gain, output, sensitivity, and gray-scale vs. color write priority settings) could have resulted in an artifactual lack of flow.

Our retrospective study did not provide adequate data to determine which patients had hepatofugal flow. Since many patients with PUV also had other varices which may have contributed as a risk for PV thrombosis, we could not determine the relative contribution of PUV towards the overall volume of portosystemic shunting. A prospective study of the effects of PUV, including measurement of blood flow velocity of the intrahepatic right and left $\mathrm{PV}$, main $\mathrm{PV}$ and patent $\mathrm{PUV}$ as well as portohepatic gradient, would provide deeper understanding. Due to retrospective design, we had small numbers of patients who had occlusive main PV thrombosis and main PV size less than $1 \mathrm{~cm}$ in both groups to allow for a sufficiently powerful statistical analysis, however trends towards increased frequency of both of these complications were observed in patients without PUV. We did not use Doppler ultrasound in most patients, but suspect that a similar relationship between paraumbilical collateral and portal thrombosis would be seen using this or other methods for evaluating the portal venous system. 
Another limitation of our study is that most examinations were only reviewed by one radiologist, although multireader review of 50 cases showed near perfect agreement regarding PV thrombosis and PUV. It is possible that one or more PUVs might have been missed, but this does not affect our observation that patients with main PV thrombosis were unlikely to have PUV.

In summary, our findings indicate that an enlarged patent PUV decreases the risk of main PV thrombosis in patients with portal hypertension who are candidates for liver transplantation.

References

1. Cahill PA, Redmond EM, Sitzmann JV (2001) Endothelial dysfunction in cirrhosis and portal hypertension. Pharmacol Ther 89:273-293

2. Price JB, Voorhees AB Jr, Britton RC (1967) Operative hemodynamic studies in portal hypertension. Arch Surg 95:843-852

3. Ohnishi K, Sato S, Pugliese D, Tsunoda T, Saito M, Okuda K (1987) Changes of splanchnic circulation with progression of chronic liver disease studied by echo-Doppler flowmetry. Am J Gastroenterol 82:507-511

4. Zironi G, Gaiani S, Fenyves D, Rigamonti A, Bolondi L, Barbara L (1992) Value of measurement of mean portal flow velocity by Doppler flowmetry in the diagnosis of portal hypertension. Hepatology 16:298-303

5. Okazaki K, Miyazaki M, Onishi S, Ito K (1986) Effect of food intake and various extrinsic hormones on portal blood flow in patients with liver cirrhosis demonstrated by pulsed Doppler with the Octoson. Scand J Gastroenterol 
$21: 1029-1038$

6. Lerut J, Tzakis AG, Bron K, et al. (1987) Complications of venous reconstruction in human orthotopic liver transplantation. Ann Surg 205:404414

7. Stieber AC, Zetti G, Todo S, et al. (1991) The spectrum of portal vein thrombosis in liver transplantation. Ann Surg 213:199-206

8. Grant EG, Tessler FN, Gomez AS, et al. (1990) Color Doppler imaging of portosystemic shunts. AJR 154:393-397

9. Kreft B, Strunk H, Flacke S, Wolff M, et al. (2000) Detection of thrombosis in the portal venous system: comparison of contrast-enhanced MR angiography with intraarterial digital subtraction angiography. Radiology 216:86-92

10. Lewis WD, Finn JP, Jenkins RL, Carretta M, Longmaid HE, Edelman RR (1993) Use of magnetic resonance angiography in the pretransplant evaluation of portal vein pathology. Transplantation 56:64-68

11. Juttner HU, Jenny JM, Ralls PW, Goldstein LI, Reynolds TB (1982) Ultrasound demonstration of portosystemic collaterals in cirrhosis and portal hypertension. Radiology 142:459-463

12. Kane RA, Katz SG (1982) The spectrum of sonographic findings in portal hypertension: a subject review and new observations. Radiology 142:453458

13. Shrout PE, Fleiss JL (1979) Intraclass correlations: uses in assessing rater reliability. Psychol Bull 86:420-429

14. Cherqui D, Duvoux C, Rahmouni A, et al. (1993) Orthotopic liver 
transplantation in the presence of partial or total portal vein thrombosis:

problems in diagnosis and management. World J Surg 17:669-674

15. Gibson RN, Gibson PR, Donlan JD, Clunie DA (1989) Identification of a patent paraumbilical vein by using Doppler sonography: importance in the diagnosis of portal hypertension. AJR 153:513-516

16. Ohhira M, Ohta H, Ohhira M, et al. (1998) Altered intrahepatic pathway of para-umbilical vein in portal hypertension. J Gastroenterol Hepatol 13:691695

17. Saddekni S, Hutchinson DE, Cooperberg PL (1982) The sonographically patent umbilical vein in portal hypertension. Radiology 145:441-443

18. Shapiro RS, Stancato-Pasik A, Glajchen N, et al. (1998) Color Doppler applications in hepatic imaging. Clin Imaging 22:272

19. Niederau C, Sonnenberg A, Muller JE, et al. (1983) Sonographic measurements of the normal liver, spleen, pancreas, and portal vein. Radiology 149:537-540

20. Webb U, Berger LA, Sherlock S (1977) Grey-scale ultrasonography of portal vein. Lancet 2:675-677

21. Weinreb J, Kumari Phillips S. C, Pochaczevsky R (1982) Portal vein measurement by real-time sonography. AJR 139:497-499

22. Seu P, Shackleton CR, Shaked A, et al. (1996) Improved results of liver transplantation in patients with portal vein thrombosis. Arch Surg 131:840844

23. Okuda K, Ohnishi K, Kimura K, et al. (1985) Incidence of portal vein 
thrombosis in liver cirrhosis. An angiographic study in 708 patients. Gastroenterology 89:279-286

24. Boyer JL, Sen Gupta KP, Biswas SK, et al. (1967) Idiopathic portal hypertension. Comparison with the portal hypertension of cirrhosis and extrahepatic portal vein obstruction. Ann Intern Med 66:41-68

25. Belli L, Romani F, Sansalone CV, Aseni P, Rondinara G (1986) Portal thrombosis in cirrhotics. A retrospective analysis. Ann Surg 203:286-291

26. Wanless IR, Wong F, Blendis LM, et al. (1995) Hepatic and portal vein thrombosis in cirrhosis: possible role in development of parenchymal extinction and portal hypertension. Hepatology 21:1238-1247

27. Wachsberg RH, Bahramipour P, Sofocleous CT, Barone A (2002) Hepatofugal flow in the portal venous system: pathophysiology, imaging findings, and diagnostic pitfalls. Radiographics 22:123-140

28. Ohnishi K, Nakayama T, Nomura F, Koen H, Okuda K (1984) Reduction of portal vein pressure with the enlargement of portal systemic shunts: observation made in one patient. J Clin Gastroenterol 6:447-451

29. Takayasu K, Takashi M, Musha H, et al. (1982) Spontaneous reversal of portal blood flow demonstrated by percutaneous transhepatic catheterization: report of two cases. Gastroenterology 82:753-757

30. Nonami T, Yokoyama I, Iwatsuki S, et al. (1992) The incidence of portal vein thrombosis at liver transplantation. Hepatology 16:1195-1198

31. Sacerdoti D, Bolognesi M, Bombonato G, Gatta A (1995) Paraumbilical vein patency in cirrhosis: effects on hepatic hemodynamics evaluated by Doppler 
sonography. Hepatology 22:1689-1694

32. Moreno AH, Burchell AR, Rousselot LM, et al. (1967) Portal blood flow in cirrhosis of the liver. J Clin Invest 46:436-445

33. Tessler FN, Gehring BJ, Gomes AS, et al. (1991) Diagnosis of portal vein thrombosis: value of color Doppler imaging. AJR 157:293-296 


\section{TABLES \& FIGURES}

Table 1. Summary of 309 patients with cirrhosis

\begin{tabular}{llll} 
Findings & $\begin{array}{l}\text { Patients with } \\
\text { PUV Group 1 } \\
(n=119)\end{array}$ & $\begin{array}{l}\text { Patients with no } \\
\text { PUV Group 2 } \\
(\mathrm{n}=190)\end{array}$ & $\begin{array}{l}\text { P } \\
\text { value }\end{array}$ \\
\hline $\begin{array}{l}\text { Overall main } \\
\text { PV thrombosis }\end{array}$ & 1 & 12 & $<0.05$ \\
$\begin{array}{l}\text { Occlusive main } \\
\text { PV thrombosis }\end{array}$ & 0 & 6 & NS \\
$\begin{array}{l}\text { Small main PV }(<1 \\
\text { cm })\end{array}$ & 3 & 10 & NS \\
Main PV diametera & $13.4 \pm 3.0$ & $11.3 \pm 3.6$ & $<0.01$ \\
$\begin{array}{l}\text { Splenorenal shunts } \\
\begin{array}{l}\text { Esophageal varices } \\
\text { Data are the number of patients }\end{array}\end{array} \quad 5$ & 19 & $<0.05$ \\
$\begin{array}{l}\text { NS, Not significant } \\
\text { a Data are mean } \pm \text { SD }\end{array}$ & 31 & NS
\end{tabular}


Figure 1. Forty-eight-year-old man with chronic portal vein (PV) thrombosis. Axial T1-weighted 3D gadolinium-enhanced gradient-echo dynamic MR images (TR/TE, 6/2.0) obtained during portal venous phase reveals $(A)$ portal venous occlusion with cavernous transformation of the PV (arrow) at the porta hepatis (B) no evidence of patent paraumblical vein in the falciform ligament (arrow). Signs of portal hypertension splenomegaly and portosystemic collateral vessels are evident.
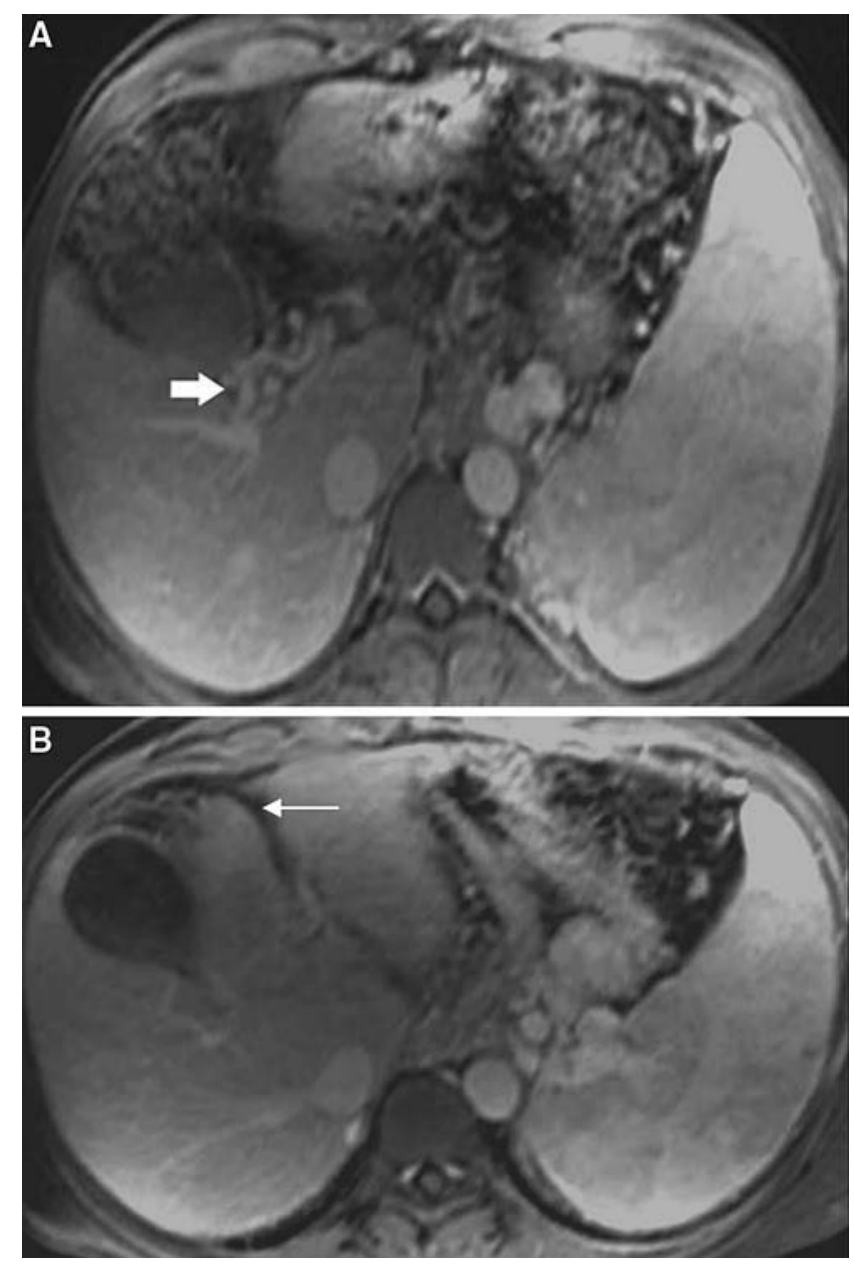
Figure 2. Patent paraumblical vein (PUV) and portal vein (PV) thrombosis in 61year-old man with portal hypertension. Axial T1-weighted 3D gadolinium-enhanced gradient-echo dynamic MR image (TR/TE, 5/1.6) obtained during portal venous phase reveals patent PUV (white arrow) and nonocclusive thrombus in the main PV (black arrow) at the portal confluence. A moderate amount of ascites is present.

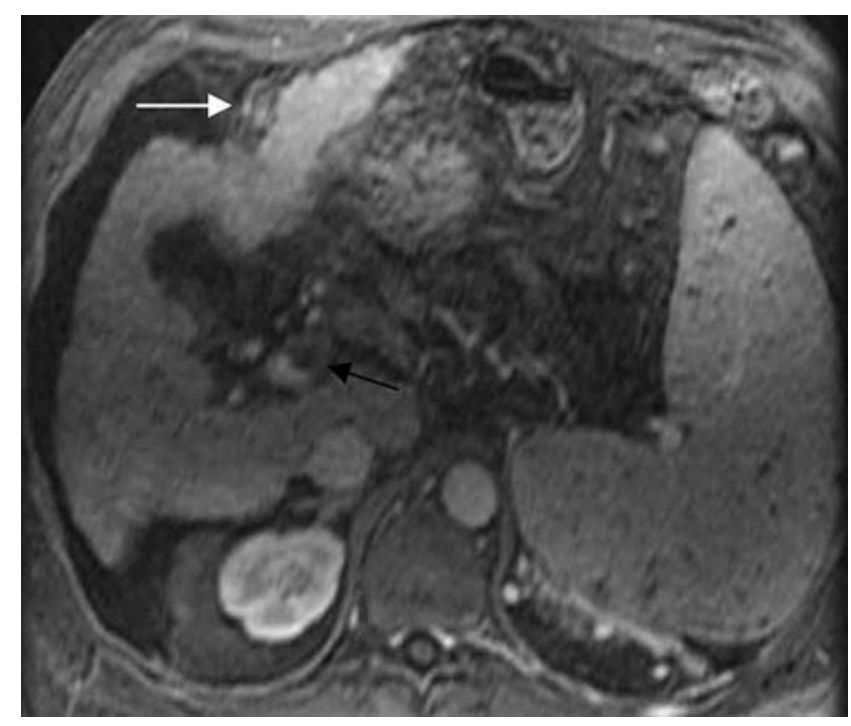


Figure 3. Patent paraumblical vein (PUV) in a 60-year-old woman with portal hypertension. Axial T1-weighted 3D gadolinium-enhanced gradient-echo dynamic MR image (TR/TE, 6/2.0) obtained during portal venous phase reveals patent intrahepatic portion of PUV with a diameter of $11 \mathrm{~mm}$ (white arrow) and normal intensely enhancing main portal vein (black arrow) (14 $\mathrm{mm}$ in diameter).

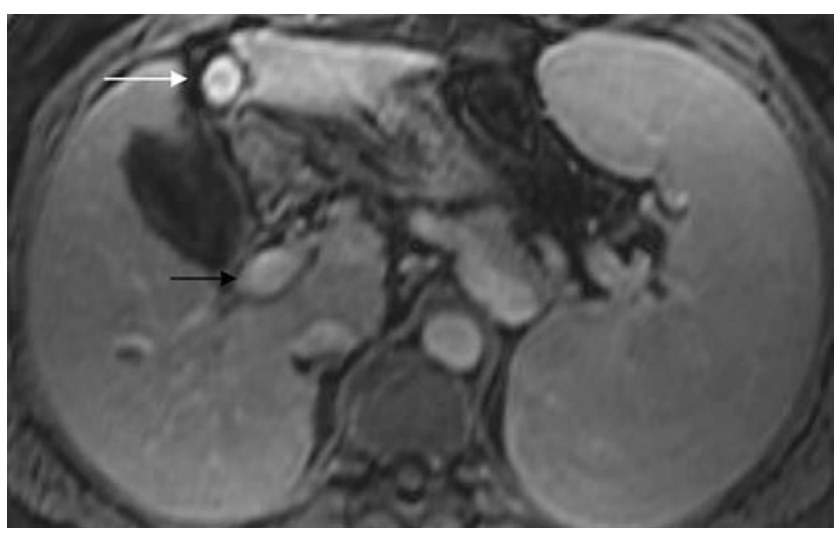


Figure 4. Patent paraumblical vein (PUV) and splenorenal portosystemic shunt in a 44-year-old man with portal hypertension. Axial T1-weighted 3D gadoliniumenhanced gradientecho dynamic MR image (TR/TE, 5/1.6) obtained during portal venous phase shows $(A)$ small $(8 \mathrm{~mm})$ main portal vein (white arrow) and prominent splenorenal collateral vessel (black arrow). The decreased signal-intensity area seen within the portal vein is due to the flow artifact. (B) Axial T1weighted 3D gadoliniumenhanced gradient-echo dynamic MR image (TR/TE, 5/1.6) obtained during portal venous phase reveals patent PUV (white arrow) and prominent splenorenal collateral vessel (black arrow).
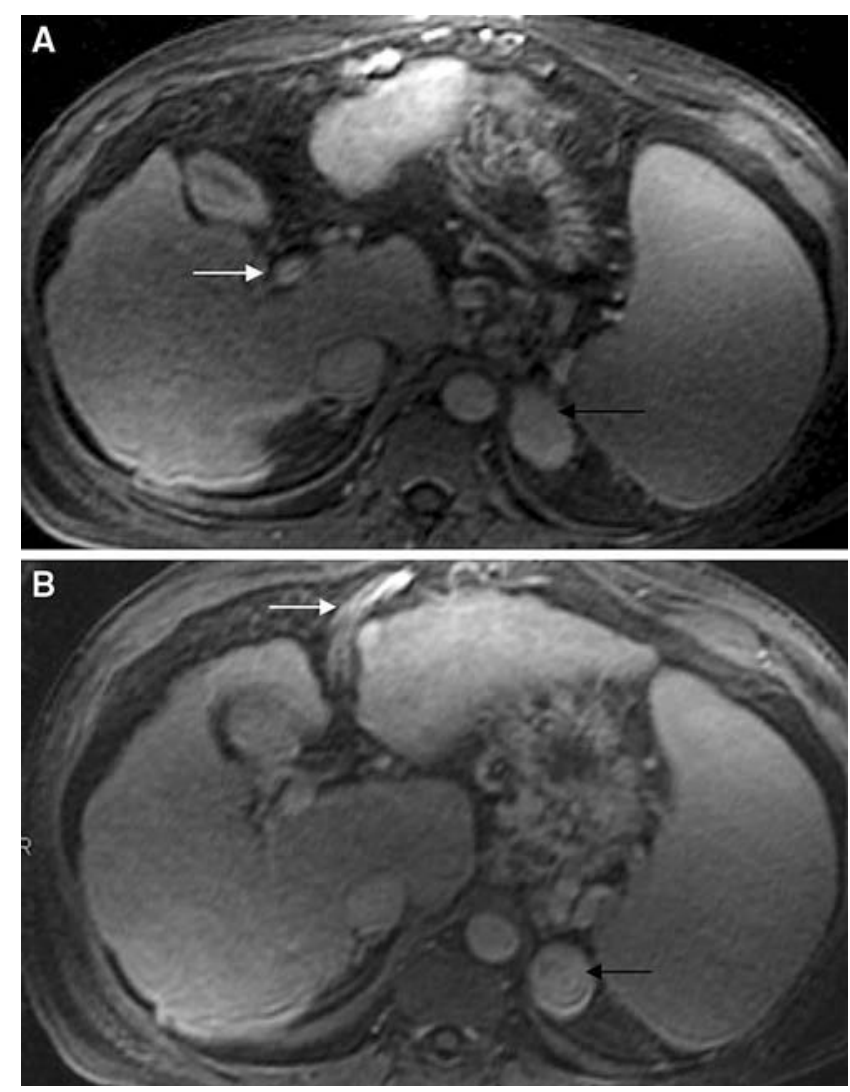